\title{
Sorção de Água em Compósitos de Poliéster Insaturado Reforçados com Tecido de Juta e Juta/Vidro: Modelagem, Simulação e Experimentação
}

\author{
Wilma S. Cavalcanti, Antonio G. B. de Lima \\ Unidade Acadêmica de Engenharia Mecânica, UFCG \\ Laura H. de Carvalho \\ Unidade Acadêmica de Engenharia de Materiais, UFCG
}

\begin{abstract}
Resumo: Estudos de compósitos reforçados com fibras naturais mostram que as dificuldades associadas ao uso de fibras vegetais como reforço em compósito de polímeros são freqüentemente relacionadas à absorção de umidade. A presença de umidade leva à impregnação e adesão interfacial fibra-matriz imperfeitas, que geram tensões internas, porosidade e falha prematura do sistema. Este problema pode ser reduzido usando fibra híbrida tramada ou tecidos tricotados. Neste sentido, este trabalho tem como objetivo estudar numérica e experimentalmente a sorção de água em compósitos de juta e juta/ vidro. Os compósitos de poliéster insaturados moldados por compressão e reforçados com tecido de juta e tecido híbrido de juta-vidro têm composições em peso de: a) $26 \%$ de juta/74\% poliéster insaturado; e b) $26 \%$ juta/32\% vidro/42\% poliéster insaturado, e dimensões de 20,00 $\times 20,00 \times 2,30 \mathrm{~mm}^{3}$ e 20,00 $\times 20,00 \times 2,82 \mathrm{~mm}^{3}$, respectivamente. Um modelo matemático tridimensional de difusão transiente foi desenvolvido para predizer a transferência de massa durante a absorção de água. A formulação matemática foi resolvida numericamente usando o método de volumes finitos e um esquema de interpolação totalmente implícito. Os resultados da distribuição do teor de umidade dentro destes compósitos e cinética de absorção de água são mostrados e analisados. O conhecimento da distribuição de umidade permite verificar áreas mais favoráveis a problemas de delaminação devido à fraqueza da interface fibra-matriz e, por conseguinte redução nas propriedades mecânicas.
\end{abstract}

Palavras-chave: Tecido híbrido, compósito, fibra vegetal, sorção de água, volumes finitos.

\section{Water Sorption in Unsaturated Polyester Composites Reinforced With Jute and Jute/ Glass Fiber Fabrics: Modeling, Simulation and Experimentation}

\begin{abstract}
Studies on composites reinforced with natural fibers show that the difficulties associated to the use of the vegetable fibers as reinforcement in polymer composites are frequently related to moisture absorption. The presence of moisture leads to imperfect impregnation and weak fiber-matrix interfacial adhesion, leading to the appearance of internal tensions, porosity, and premature failure of the system. This problem can be reduced by employing hybrid fiber weaved or knitted fabrics. This work is aimed at studying water sorption in composites of jute and jute/glass. The compression molded unsaturated polyester composites reinforced with jute fabric and a hybrid jute-glass fabric had the following weight compositions: a) $26 \%$ jute $/ 74 \%$ unsaturated polyester; and b) $26 \%$ jute/32\% glass roving/42\% unsaturated polyester and dimensions of $20.00 \times 20.00 \times 2.30 \mathrm{~mm}^{3}$ and $20.00 \times 20.00 \times 2.82 \mathrm{~mm}^{3}$, respectively. A three-dimensional transient diffusion mathematical model was developed to predict mass transfer during water absorption. The mathematical formulation was numerically solved using a finite-volume method and an implicit interpolation scheme. The results of moisture content distribution inside these composites and water absorption kinetics are shown and analyzed. The knowledge of this moisture distribution allows verifying more favorable areas for delamination due to the weakness of the fiber-matrix interface and consequently reduction in the mechanical properties.
\end{abstract}

Keywords: Hybrid fabric, composite, vegetable fiber, water sorption, finite-volume.

\section{Introdução}

Nos últimos anos houve um crescimento rápido no uso de fibras vegetais renováveis como reforços em matérias compósitos. Plásticos reforçados feito com materiais celulósicos são de baixo custo, leves, melhoram as propriedades mecânicas, e estão livres de risco à saúde.

Apesar da atração pelo uso de compósitos de materiais poliméricos reforçados com fibras vegetais, eles exibem

Autor para correspondência: Laura H. de Carvalho, Centro de Ciências e Tecnologia, Unidade Acadêmica de Engenharia de Materiais, Av. Aprígio Veloso, 882, Bodocongó, CEP: 58429-900, Caixa Postal 10034, Campina Grande, PB, Brasil. E-mail: laura@dema.ufcg.edu.br 
baixas propriedades mecânicas e resistência à umidade quando comparados com compósitos reforçados com fibras sintéticas, e, portanto, são muito sensíveis às influências de agentes ambientais tais como água ${ }^{[1]}$. A presença de umidade na interface fibra-matriz induz formação de vazios na interface. A umidade interfacial também contribui para o inicio do processo de delaminação entre a fibra e a resina curada $^{[2]}$. O enfraquecimento interfacial dos compósitos com fibras vegetais pode ser melhorado através do uso de reforços híbridos, ou na combinação de laminados ou ainda através de misturas de fibras ${ }^{[3]}$. A hibridização de fibras vegetais com fibras mais fortes tais como fibras de vidro, também pode melhorar a resistência à umidade dos compósitos. A vantagem de usar compósitos híbridos que contenham dois ou mais diferentes tipos de fibras, é que um tipo de fibras pode complementar o que falta na outro. Como consequiência o balanço no desempenho e no custo pode ser encontrado através do próprio design ${ }^{[1]}$.

Vários trabalhos sobre a cinética de absorção de água e/ou umidade em sistemas compósitos apresentando soluções analíticas e/ou numéricas foram encontrados na literatura. No entanto, todos os trabalhos se resumem a considerar a difusão de líquido no interior do sólido como sendo unidimensiona ${ }^{[4-15]}$. Em alguns casos para levar em consideração a tridimensionalidade do problema alguns pesquisadores têm utilizado soluções analíticas para o problema tridimensional aplicada para tempos longos, o que simplifica bastante o problema $^{[16-18]}$.

Do exposto, o objetivo deste trabalho é usar uma modelagem matemática tridimensional transiente para predizer a transferência de massa durante a absorção de água em compósitos usando uma formulação matemática resolvida numericamente pelo método de volumes finitos e um esquema de interpolação totalmente implícito. Os compósitos sobre investigação são à base de poliéster insaturado com tecidos de juta e juta-vidro.

\section{Experimental}

O poliéster insaturado usado neste trabalho foi fornecido pela Resana S.A. e curado com 1\% de metil-etil-cetona (MEK) fornecido pelo VI Fiberglass. A juta (10/2) e a fibra de vidro (111A-408) foram fornecidos pelas Cia. Têxtil Castanhal PA. e Owens Corning do Brasil, respectivamente.

$\mathrm{O}$ tecido de juta foi fabricado em tear manual, utilizando fios de juta (10/2). Utilizou-se um pente 18 e 1 fio por pua, e 1 batida aberta e uma fechada. $\mathrm{O}$ tecido assim tramado apresentou 42 fios $/ 10 \mathrm{~cm}$ no urdume e 39 fios $/ 10 \mathrm{~cm}$ na trama (Figura 1a).

O tecido híbrido juta/vidro foi fabricado em tear manual, contendo cordões de juta (10/2) no urdume e mechas de fibra de vidro na trama. Devido à espessura reduzida das mechas de vidro, foram lançadas duas mechas em cada passada. Utilizou-se um pente 18 e 1 fio por pua, e 1 batida aberta e uma fechada. $\mathrm{O}$ tecido assim tramado apresentou 40 fios $/ 10 \mathrm{~cm}$ no

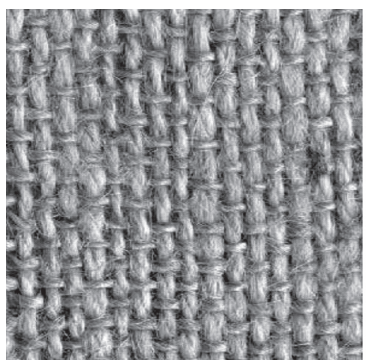

(a)

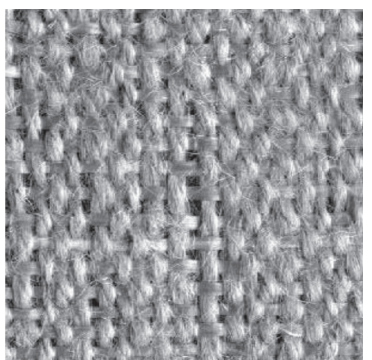

(b)
Figura 1. Aparência dos tecidos de a) juta; e b) juta/vidro empregados neste trabalho.

urdume e 35-38 fios/10 cm na trama. A composição do tecido híbrido utilizado foi de $44 \%$ de juta e $56 \%$ de vidro em massa (Figura 1b).

Os compósitos com variados teores de fibras foram moldados por compressão. Os tecidos foram cortados de acordo com as dimensões do molde $(170 \times 140 \mathrm{~mm})$, préimpregnados com resina e colocado no molde. $\mathrm{O}$ excesso de resina foi vertido em cima dos tecidos, e o molde foi mantido fechado sob carga de 8 t por 8 horas. Foram fabricadas placas de compósitos de $\left(140 \times 170 \mathrm{~mm}^{2}\right)$ com 2 camadas de tecido de juta ( $26 \%$ de juta/74\% de poliéster insaturado) e com 4 camadas de tecidos híbridos juta/vidro (26\% de juta/32\% de vidro/42\% de poliéster insaturado). Destas placas, foram serradas amostras com dimensões de $20,00 \times 20,00 \times 2,30 \mathrm{~mm}^{3}$ (juta/poliester insaturado) e de $20,00 \times 20,00 \times 2,82 \mathrm{~mm}^{3}$ (juta/vidro/poliéster insaturado); suas arestas destas amostras foram seladas com resina e secadas até atingir peso constante, em uma estufa de circulação de ar, operando a $105{ }^{\circ} \mathrm{C}$. Imediatamente depois de secas as amostras foram pesadas e em seguidas imersas em água destilada à temperatura ambiente. Em tempos pré-determinados as amostras eram retiradas da água, secas rapidamente com papel ou tecido de baixa absorção e pesadas em balança analítica, com precisão de $0,1 \mathrm{mg}$. O teor de água absorvido foi calculado comparando-se a massa do sólido após exposição a água e a sua massa inicial (totalmente seca).

\section{Modelagem Matemática}

Para descrever a transferência de massa no sólido com forma de paralelepípedo (Figura 2), as seguintes considerações são admitidas:

- o sólido é homogêneo e isotrópico;

- o efeito de capilaridade é desprezível;

- existe simetria no centro do sólido;

- a condição de contorno é de equilíbrio na superfície do sólido;

- a densidade do sólido é considerada constante;

- nenhuma geração de massa no interior do sólido ocorre; e

- o sólido é considerado totalmente seco no inicio do processo. 


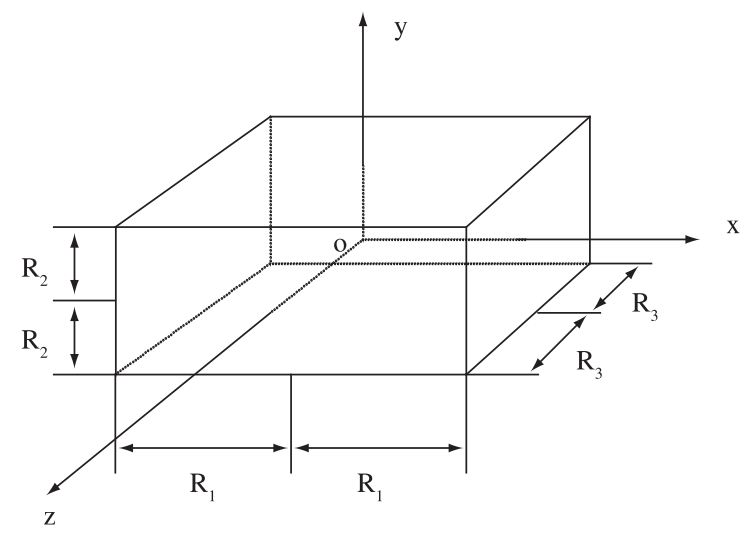

Figura 2. Configuração geométrica do problema físico.

A Figura 2 ilustra um sólido paralelepípedo de dimensões $2 R_{1} \times 2 R_{2} \times 2 R_{3}$. Para este caso, a equação diferencial geral que descreve o fenômeno de difusão é da Equação 1:

$$
\frac{\partial \mathrm{M}}{\partial \mathrm{t}}=\nabla \cdot(\mathrm{D} \nabla \mathrm{M})
$$

onde $\mathrm{D}$ é o coeficiente de difusão, $\mathrm{M}$ é o teor de umidade e $\mathrm{t}$ é o tempo de processo.

Devido a simetria do problema foi considerado $1 / 8$ do volume do sólido. As condições inicial, de simetria e de contorno são as seguintes (Equação 2, 3, 4 e 5):

$\Rightarrow$ Condição inicial

$\mathrm{M}(\mathrm{x}, \mathrm{y}, \mathrm{z}, \mathrm{t}=0)=\mathrm{M}_{\mathrm{o}}, \quad 0<\mathrm{x}<\mathrm{R}_{1}$,

$0<\mathrm{y}<\mathrm{R}_{2}, \quad 0<\mathrm{z}<\mathrm{R}_{3}$

$\Rightarrow$ Condição de simetria

$$
\begin{aligned}
& \frac{\partial M(x=0, y, z, t)}{\partial x}=\frac{\partial M(x, y=0, z, t)}{\partial y}= \\
& \frac{\partial M(x, y, z=0, t)}{\partial x}=0, t>0
\end{aligned}
$$

$\Rightarrow$ Condições de contorno na superficie:

$$
\begin{aligned}
& M\left(x=R_{1}, y, z, t\right)=M\left(x, y=R_{2}, z, t\right)= \\
& M\left(x, y, z=R_{3}, t\right)=M_{e}, t>0
\end{aligned}
$$

O teor de umidade médio foi obtido por:

$$
\overline{\mathrm{M}}=\frac{1}{\mathrm{~V}} \int_{\mathrm{V}} \mathrm{MdV}
$$

Neste trabalho, o coeficiente de difusão foi considerado dependente do teor de umidade do sólido de acordo com a seguinte Equação 6.

$$
\mathrm{D}=\mathrm{D}_{\mathrm{o}} \operatorname{Exp}(\mathrm{a} \overline{\mathrm{M}})
$$

onde $\mathrm{D}_{\mathrm{o}}$ e a são constantes obtidas a partir de dados experimentais e $\overline{\mathrm{M}}$ o teor de umidade médio em base seca.

\section{Método Numérico}

Neste trabalho, o método dos volumes finitos foi usado para discretizar a equação governante. A Figura 3 representa o volume diferencial do domínio físico (Figura 2), onde os pontos nodais (W, E, N, S, F, T), as dimensões e comprimentos do volume de controle são apresentados.

Assumindo uma formulação totalmente implícita, onde os termos são estimados nos tempos $t+\Delta t$, a Equação (1) foi integrada no volume de controle da Figura 3 , que corresponde a pontos internos do domínio, e também no tempo. Como resultado, a Equação (1) foi obtido na forma discretizada utilizando a prática $\mathrm{B}$ (pontos nodais no centro do volume de controle ${ }^{[19,20]}$ e pode ser escrita na forma linear como segue (Equação 7):

$$
\begin{aligned}
& A_{P} M_{P}^{*}=A_{E} M_{E}^{*}+A_{W} M_{W}^{*}+A_{N} M_{N}^{*}+ \\
& A_{S} M_{S}^{*}+A_{T} M_{T}^{*}+A_{F} M_{F}^{*}+B
\end{aligned}
$$

onde:

$\mathrm{A}_{\mathrm{E}}=\frac{\mathrm{D}_{\mathrm{e}} \Delta \mathrm{y} \Delta \mathrm{z}}{\delta \mathrm{x}_{\mathrm{e}}} \quad \mathrm{A}_{\mathrm{W}}=\frac{\mathrm{D}_{\mathrm{w}} \Delta \mathrm{y} \Delta \mathrm{z}}{\delta \mathrm{x}_{\mathrm{w}}} \quad \mathrm{A}_{\mathrm{N}}=\frac{\mathrm{D}_{\mathrm{n}} \Delta \mathrm{z} \Delta \mathrm{x}}{\delta \mathrm{y}_{\mathrm{n}}} \quad \mathrm{A}_{\mathrm{S}}=\frac{\mathrm{D}_{\mathrm{s}} \Delta \mathrm{z} \Delta \mathrm{x}}{\delta \mathrm{y}_{\mathrm{S}}}$ $\mathrm{A}_{\mathrm{T}}=\frac{\mathrm{D}_{\mathrm{t}} \Delta \mathrm{x} \Delta \mathrm{y}}{\delta \mathrm{z}_{\mathrm{t}}} \quad \mathrm{A}_{\mathrm{F}}=\frac{\mathrm{D}_{\mathrm{f}} \Delta \mathrm{x} \Delta \mathrm{y}}{\delta \mathrm{z}_{\mathrm{f}}} \quad \mathrm{A}_{\mathrm{P}}^{\mathrm{o}}=\frac{\Delta \mathrm{x} \Delta \mathrm{y} \Delta \mathrm{z}}{\Delta \mathrm{t}} \quad \mathrm{B}=\mathrm{A}_{\mathrm{P}}^{\mathrm{o}} \mathrm{M}_{\mathrm{p}}^{* \mathrm{o}}$ $A_{P}=A_{E}+A_{W}+A_{N}+A_{S}+A_{T}+A_{F}+A_{P}^{o} \quad M^{*}=\frac{M-M_{e}}{M_{o}-M_{e}}$

onde $\mathrm{D}$ na interface do volume de controle foi calculado de acordo com a literatura ${ }^{[20]}$.

A Equação (7) é valida para cada volume de controle do domínio em estudo, o que gera um conjunto de equações algébricas lineares.

O conjunto de equações foi resolvido interativamente usando o método Gauss-Seidel. O seguinte critério de convergência foi usado (Equação 8):

$$
\left|\mathrm{M}^{* \mathrm{n}-1}-\mathrm{M}^{* \mathrm{n}}\right| \leq 10^{-8}
$$

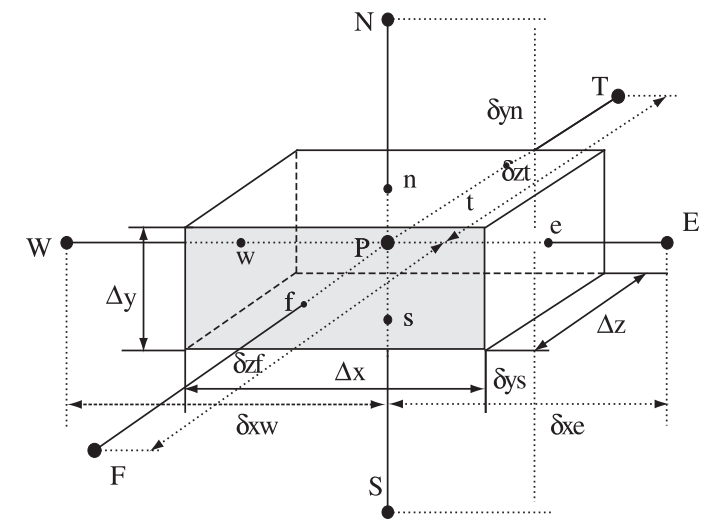

Figura 3. Volume de controle usado neste trabalho. 
onde $\mathrm{n}$ representa a n-ésima iteração em cada instante de tempo.

O coeficiente de difusão foi encontrado variando o D para minimizar a soma do desvio quadrático entre os dados experimentais e o predito. $\mathrm{O}$ desvio relativo entre os valores experimental e calculado (resíduo relativo, ERMQ) e a variância $\left(S^{2}\right)$ são definidos como seguem ${ }^{[21]}$ (Equação 9e 10):

$$
\begin{aligned}
& \text { ERMQ }=\sum_{i=1}^{n}\left(\bar{M}_{i, N u m}^{*}-\bar{M}_{i, \text { Exp }}^{*}\right)^{2} \\
& S^{2}=\frac{\text { ERMQ }}{(\hat{\mathrm{n}}-1)}
\end{aligned}
$$

onde $\hat{n}$ é o número de pontos experimentais. Os menores valores ERMQ e $\mathrm{S}^{2}$ foram usados como critério para obter os melhores valores do coeficiente de difusão.

\section{Resultados e Discussão}

Para obter resultados numéricos, desenvolveu-se um código computacional no ambiente do Mathematica ${ }^{\circledR}$ e usouse uma malha de $20 \times 20 \times 20$ pontos e $\Delta \mathrm{t}=20$ segundos. Desde que, os resultados numéricos obtidos de um determinado modelo matemático são fortemente dependente das condições de contorno, das propriedades termo-físicas da geometria, e do numero de pontos da malha e passo de tempo usado, estes resultados foram obtidos após um estudo de refino de malha e de tempo conforme descrito $\mathrm{em}^{[22]}$.

Para validar a metodologia, os resultados numéricos do teor de umidade médio dos compósitos sob investigação foram comparados com os dados experimentais, conforme mostra Figura 4. A análise desta figura indica que o modelo empregado aqui é apropriado. A modelagem conduziu a erros de $0,0234468 \mathrm{~kg} / \mathrm{kg}^{2}$ com variância de $0,000239 \mathrm{~kg} / \mathrm{kg}^{2}$ no final do processo de 600 horas, i.e., 25 dias para o compósito poliéster/juta (74/26\% em peso), e de $0,2151 \mathrm{~kg} / \mathrm{kg}^{2} \mathrm{com}$

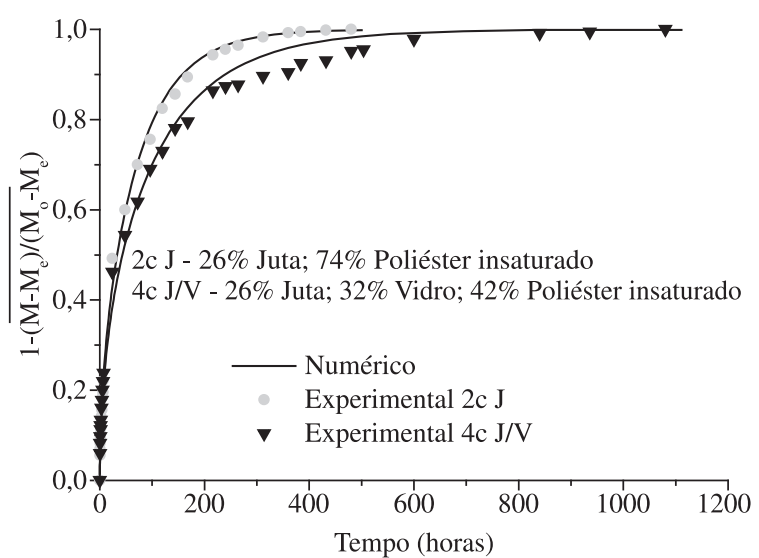

Figura 4. Comparação entre o teor de umidade médio adimensional predito e experimental obtidos durante a sorção de água dos compósitos de poliéster insaturado reforçados por juta e juta-vidro. $2 \mathrm{cJ}-2$ camadas de juta; $4 \mathrm{cJ} / \mathrm{V}-4$ camadas juta/vidro. variância de $0,00129 \mathrm{~kg} / \mathrm{kg}^{2}$ no final do processo 1248 horas, i.e., 52 dias para o compósito híbrido poliéster/juta-vidro (42/26/32\% em peso). A Figura 3 também evidencia que uma quantidade equivalente de água foi absorvida pelos compósitos com similar teor de juta, o que leva a uma indicação que a sorção de água nestes compósitos é dominada pela fibra vegetal (juta), embora a presença da fibra de vidro atue no sentido de retardar o processo. Nesta figura, a nomenclatura $2 \mathrm{cJ}$ significa duas camadas de juta, enquanto $4 \mathrm{c} \mathrm{J} / \mathrm{V}$ quer dizer quatro camadas de juta/vidro.

A distribuição do teor de umidade dos compósitos poliéster-juta e poliéster-juta-vidro nos planos $\mathrm{x}^{*}=\mathrm{x} / \mathrm{R}=0,5$ e $\mathrm{z}^{*}=\mathrm{z} / \mathrm{R}=0,5$ para o material $26 \%$ de juta/ $74 \%$ de poliéster insaturado (2c J) e $x^{*}=x / R=0,5$ para o material $26 \%$ de juta/32\% de vidro/42\% de poliéster insaturado (4c J/V) nos tempos 3, 20, 44, 133 e 208 horas está apresentada nas Figuras 5,6 e 7 respectivamente. A existência de alto gradiente de umidade na região próxima ao vértice do sólido está ilustrada. As Figuras 5, 6 e 7 mostram que o teor de umidade aumenta com o tempo de imersão. Quando comparada a Figura 5 com a Figura 6 é observado que a distribuição do teor de umidade é similar nos dois lados do sólido. Na área próxima dos pontos $\left(\mathrm{x}=\mathrm{R}_{1}, \mathrm{y}=\mathrm{R}_{2}, \mathrm{z}=\mathrm{R}_{3}\right)$ o sólido umidifica mais rapidamente.

Uma comparação entre as Figuras 5 e 7, indica que, apesar das diferenças na espessura, o processo de umidificação do compósito reforçado por juta foi mais rápido que o compósito reforçado por tecido híbrido. Este comportamento é atribuído ao aumento na relação área/volume bem como a maior afinidade à água com materiais celulósicos. Isto indica que a hibridização de fibras vegetais com sintéticas como, por exemplo, fibra de vidro, é uma maneira eficiente
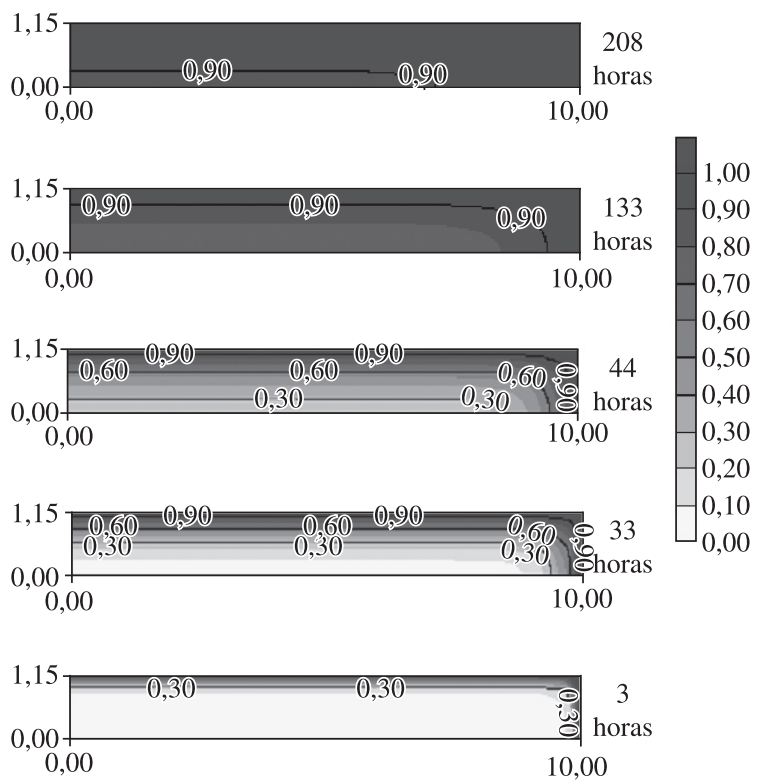

Figura 5. Perfil do teor de umidade dentro do material com $26 \%$ juta e $74 \%$ poliéster insaturado no plano $\mathrm{x}^{*}=\mathrm{x} / \mathrm{R}=0,5 \mathrm{em}$ alguns tempos de processo (Caso 2cJ). 

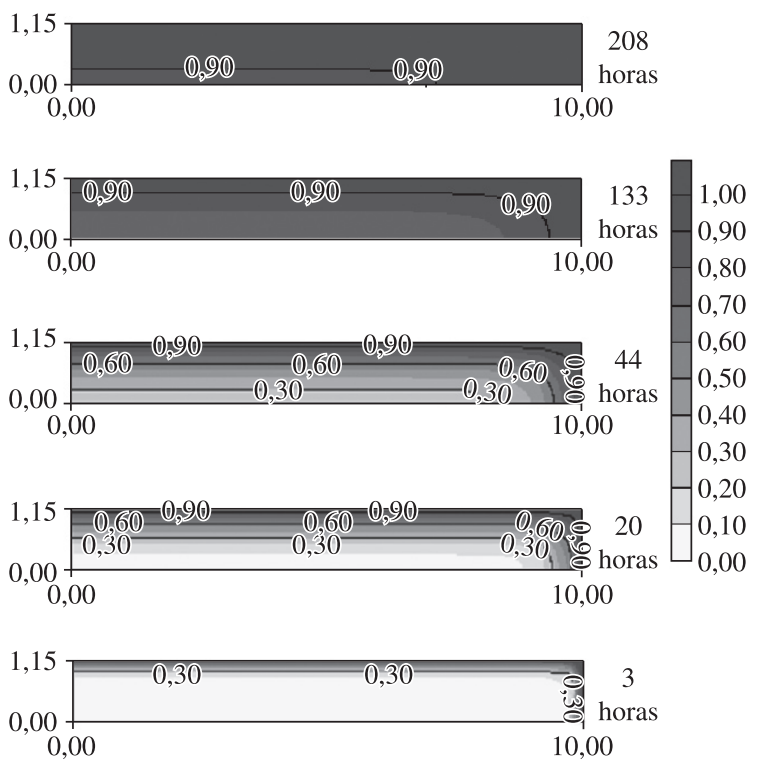

Figura 6. Perfil do teor de umidade dentro do material com $26 \%$ juta e $74 \%$ poliéster insaturado no plano $\mathrm{z}^{*}=\mathrm{z} / \mathrm{R}=0,5 \mathrm{em}$ alguns tempos de processo (Caso $2 \mathrm{cJ})$.
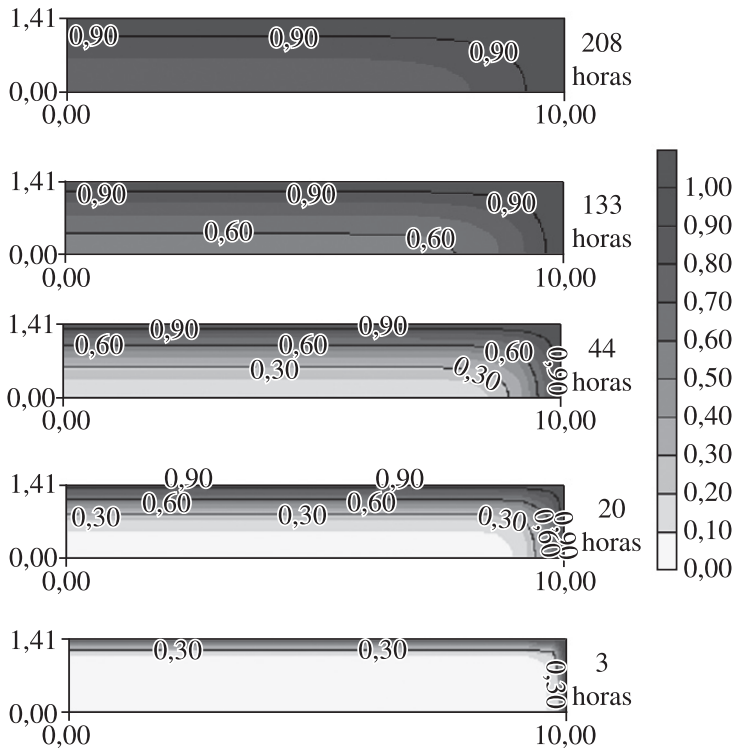

Figura 7. Perfil do teor de umidade dentro do material com $26 \%$ juta, $32 \%$ vidro e $42 \%$ poliéster insaturado no plano $x^{*}=x / R=0,5$ em alguns tempos de processo (Caso $4 \mathrm{cJ} / \mathrm{V})$.

para aumentar a resistência de ganho de água de compósitos poliméricos $^{[3]}$.

É importante conhecer o perfil e o teor de umidade dentro do material para predizer que áreas são mais sensíveis à tensão mecânica, que pode causar trincas, rupturas e deformações, e conseqüentemente diminuir a qualidade do produto ${ }^{[22]}$.

A Tabela 1 e Figura 8 mostram os valores do coeficiente de difusão para os dados obtidos. Como esperado, o coeficiente de difusão tende a diminuir com o aumento do teor de umidade do material. A difusividade de massa em função desta variável gerou dados numéricos que se ajustou muito bem aos resultados experimentais, com erros essencialmente
Tabela 1. Valores dos parâmetros da Equação (6) obtidos neste trabalho.

\begin{tabular}{ccc}
\hline \multirow{2}{*}{ Parâmetro } & \multicolumn{2}{c}{ Material } \\
\cline { 2 - 3 } & Juta-poliéster & Juta-vidro-poliéster \\
\hline Do $\left(\mathrm{m}^{2} / \mathrm{s}\right)$ & $18,0 \times 10^{-13}$ & $22,0 \times 10^{-13}$ \\
$\mathrm{a}$ & $-0,5$ & $-4,2$ \\
\hline
\end{tabular}

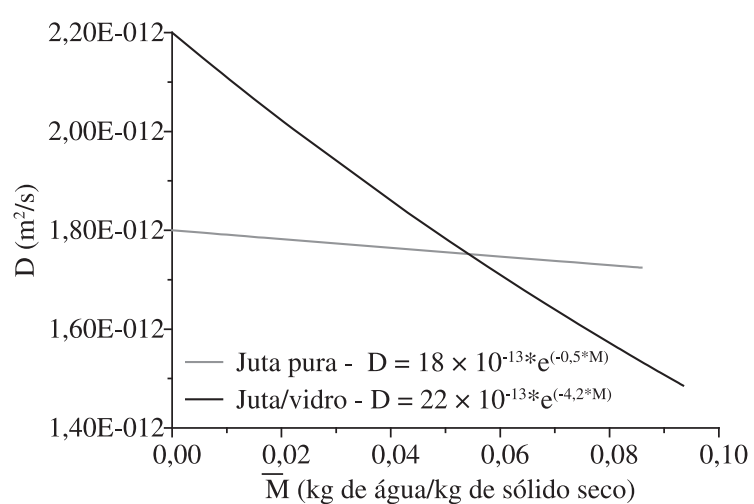

Figura 8. Evolução do coeficiente de difusão em função do teor de umidade médio do compósito poliéster reforçado por tecido de juta e juta/vidro.

melhores do que aquele obtido assumindo propriedades constantes, como utilizado por diversos pesquisadores ${ }^{[-7,14]}$. A variação deste parâmetro para todo o processo foi de $4,2 \%$ para o caso de juta pura $\left(M_{e}=0,08587 \mathrm{~kg} / \mathrm{kg}\right)$ e de $32,5 \%$ no caso de juta-vidro $\left(M_{e}=0,09346 \mathrm{~kg} / \mathrm{kg}\right)$.

\section{Conclusões}

Considerando os resultados obtidos, pode-se concluir de maneira geral que:

- O modelo matemático usado descreve adequadamente o processo de difusão de água dentro do compósito.

- Alta difusão de água foi obtida nas primeiras 50 horas de sorção, e a taxa de difusão de água diminui para tempos mais longos de imersão.

- As regiões vizinhas aos vértices do sólido apresentam as maiores taxas de transferência de massa.

\section{Agradecimentos}

Os autores agradecem a Resana S.A., Cia Têxtil Castanhal, Owens-Corning do Brasil e VI Produtos Químicos pela doação dos insumos, a José Leandro Gomes da Silva e ao Lar do Garoto pela fabricação dos tecidos; ao CNPq/PADCT e FINEP pelo suporte financeiro, ao CNPq pela bolsa (DCR e $\mathrm{PQ}$ ), e aos pesquisadores referenciados que com seus estudos ajudaram no melhoramento deste trabalho.

\section{Referências Bibliográficas}

1. Thwe, M. M. \& Liao, K. - Compos. Appl. Sci. Manuf., 33, p.43 (2002).

2. Mohanty, A. K. \& Misra, M. - Polym. Plast. Tech. Eng., 34, p.729 (1995). 
3. Cavalcanti, W. S.; Carvalho, L. H. \& Lima, A. G. B. Rev. Mat., 10, p.479 (2005).

4. Apicella, A.; Miglaresi, C.; Nicodema, L.; Nicolais, L.; Iaccarino, L. \& Roccotelli, S. - Compos., 13, p.406 (1982)

5. Han, K. S. \& Koutsky, J. - Compos., 14, p.67 (1983).

6. Ellis, B. E. \& Found, M. S. - Compos., 14, p.237 (1983).

7. Camino, G.; Luda, M. P.; Polishchuk, A. Ya; Revellino, M.; Blancon, R.; Merle, G. \& Martinez-Vega, J. J. Compos. Sci. Technol., 57, p.1469 (1997).

8. Marcovich, N. E.; Reboredo, M. M. \& Aranguren, M. I. - Polymer, 40, p.7313 (1999).

9. Choi, H. S.; Ahn, K. J.; Nan, J. D. \& Chun, H. J. Compos. Appl. Sci. Manuf., 32, p.709 (2001).

10. Srihari, S.; Revathi, A. \& Rao, R. M. V. G. K. - J. Reinf. Plast. Compos., 21, p.983 (2002).

11. Katzman, H. A.; Castaneda, R. M. \& Lee, H. S. - Compos. Appl. Sci. Manuf., 39, p.887 (2008).

12. Yao, J. \& Ziegmann, G. - J. Compos. Mat., 41, p.993 (2007).

13. Panthapulakkal, S. \& Sain, M. - J. Compos. Mat., 41, p.1871 (2007).

14. Najafi, K. S.; Kiaefar, A.; Hamidinia, E. \& Tajvidi, M. J. Reinf. Plas. Compos., 26, p.341 (2007).
15. Czél, G. \& Czigány, T. A. - J. Compos. Mat., 42, p.2815 (2008).

16. Chateauminois, A.; Vicent, L.; Chabert, B. E. \& Soulier, J. P. - Polym., 35, p.4766 (1994).

17. Pavan, R. M. V.; Saravanan, V.; Dinesh, A. R.; Rao, Y. J.; Srihari, S. \& Revathi, A. - J. Reinf. Plast. Compos., 20, p.1036 (2001).

18. Bao, L. R. \& Yee, A. F. - Compos. Sci. Technol., 62, p.2099 (2002).

19. Maliska, C. R. - "Computational Heat Transfer and Fluid Mechanics", LTC, Rio de Janeiro (2004).

20. Patankar, S. V. - "Numerical heat transfer and fluid flow", Hemisphere Publishing Corporation, New York (1980)

21. Figliola, R. S. \& Beasley, D. E. - "Theory and design for mechanical measurements", John Wiley \& Sons, New York (1995).

22. Nascimento, J. J. S. - "Fenômenos de difusão transiente em sólidos paralelepípedos. Estudo de caso: secagem de materiais cerâmicos". Tese de Doutorado, Universidade Federal da Paraíba, Brasil (2002).

Enviado: 11/08/09

Reenviado: 30/11/09

Aceito: $15 / 12 / 09$

DOI: $10.1590 / \mathrm{S} 0104-14282010005000007$ 